In the format provided by the authors and unedited.

\title{
The marine fish food web is globally connected
}

\author{
Camille Albouy $\oplus^{1 \star}$, Philippe Archambaultt $\oplus^{2}$, Ward Appeltans ${ }^{3}$, Miguel B. Araújo $\circledast^{4,5,6}$, \\ David Beauchesne ${ }^{7}$, Kevin Cazelles $\oplus^{8}{ }^{8}$, Alyssa R. Cirtwill $\oplus^{9,20}$, Marie-Josée Fortin ${ }^{10}$, Nuria Galianaa", \\ Shawn J. Leroux ${ }^{12}$, Loïc Pellissier ${ }^{13,14}$, Timothée Poisot ${ }^{15,16}$, Daniel B. Stouffer ${ }^{{ }^{9}}{ }^{9}$, Spencer A. Wood ${ }^{17,18}$ \\ and Dominique Gravel ${ }^{15,19}$
}

IIFREMER, unité Ecologie et Modèles pour l'Halieutique, Nantes, France. ²Departement de biologie, Faculté des sciences et de génie, Université Laval, Québec, Québec, Canada. ${ }^{3}$ Ocean Biogeographic Information System, IODE, Intergovernmental Oceanographic Commission, UNESCO, Ostend, Belgium. ${ }^{4}$ Departmento de Biogeografía y Cambio Global, Museo Nacional de Ciencias Naturales, Consejo Superior de Investigaciones Científicas, Madrid, Spain. ${ }^{5}$ InBio/Centro de Investigação em Biodiversidade e Recursos Genéticos, Universidade de Évora, Évora, Portugal. ${ }^{6}$ Center for Macroecology, Evolution, and Climate, Natural History Museum of Denmark, University of Copenhagen, Copenhagen, Denmark. ${ }^{7}$ Institut des sciences de la mer, Université du Québec à Rimouski, Rimouski, Québec, Canada. ${ }^{8}$ Department of Integrative Biology, University of Guelph, Guelph, Ontario, Canada. ${ }^{9} \mathrm{Centre}$ for Integrative Ecology, School of Biological Sciences, University of Canterbury, Christchurch, New Zealand. ${ }^{10}$ Department of Ecology and Evolutionary Biology, University of Toronto, Toronto, Ontario, Canada. "Centre for Biodiversity Theory and Modelling Station d'Ecologie Théorique et Expérimentale du CNRS, Moulis, France. ${ }^{12}$ Department of Biology, Memorial University, St. John's, Newfoundland, Canada. ${ }^{13}$ Landscape Ecology, Institute of Terrestrial Ecosystems, Department of Environmental Systems Science, ETH Zürich, Zürich, Switzerland. ${ }^{14}$ Swiss Federal Research Institute WSL, Birmensdorf, Switzerland. ${ }^{15}$ Département des Sciences Biologiques, Université de Montréal, Montréal, Québec, Canada. ${ }^{16}$ Québec Centre for Biodiversity Sciences, McGill University, Montréal, Québec, Canada. ${ }^{17}$ EarthLab, University of Washington, Seattle, WA, USA. ${ }^{18}$ eScience Institute, University of Washington, Seattle, WA, USA. ${ }^{19}$ Département de Biologie, Universite de Sherbrooke, Sherbrooke, Québec, Canada. ${ }^{20}$ Present address: Department of Ecology, Evolution, and Plant SciencesPhysics, Chemistry and Biology (IFM), Linköping Stockholm University, Stockholm, Sweden. *e-mail: albouycamille@gmail.com 


\section{Supplementary Materials for}

The marine fish food web is globally connected

Camille Albouy, Philippe Archambault, Ward Appeltans, Miguel B. Araújo, David Beauchesne, Kevin Cazelles, Alyssa R. Cirtwill, Marie-Josée Fortin, Nuria Galiana, Shawn J. Leroux, Loïc Pellissier, Timothée Poisot, Daniel B. Stouffer, Spencer A. Wood, Dominique Gravel.

Correspondence to: albouycamille@gmail.com

This PDF file includes:

Figs. S1 to S10

Tables S1 to S5 
Fig. S1 | Distribution of the 13,916,517 Ocean Biogeographic System (OBIS) (16) occurrences (a) used for the construction of the 11,365 marine fish distribution maps species across the world and averaged across latitudes (b). The black bars on the chart represent the standard error of the mean. The blue line represents a GAM model fitted between the mean number of OBIS occurences $(\log )$ and the latitude $\left(b\right.$; adjusted $\mathrm{R}^{2}=0.7$, estimated degrees of freedom $=8.8$ ) within shaded grey the $95 \%$ confidence limits. The coastline was defined by using the Global Selfconsistent, Hierarchical, High-resolution Geography Database (51).

a

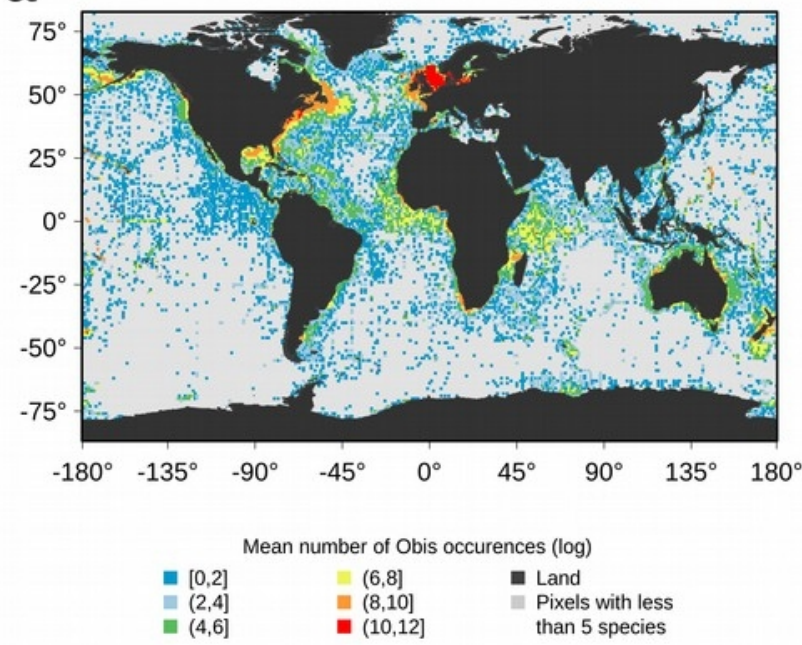

b

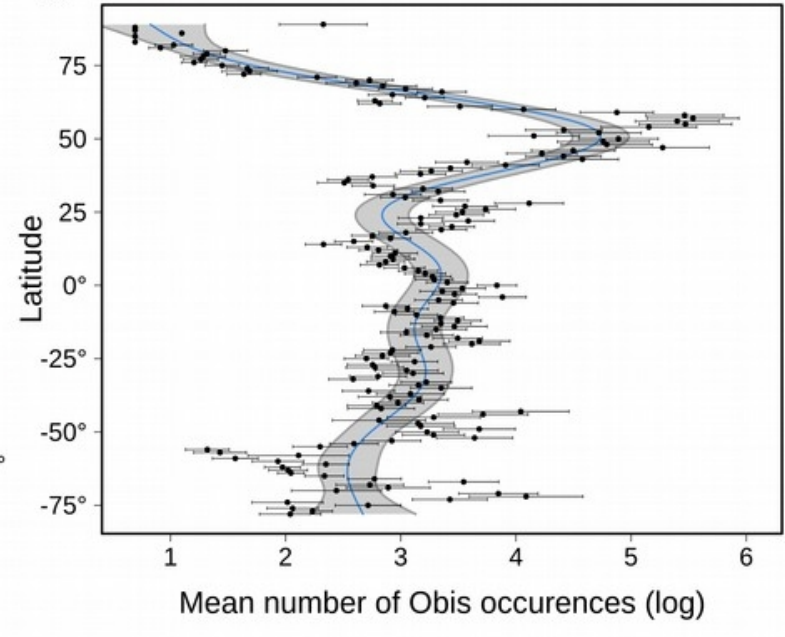


Fig S2 | Evaluation of the calibration process. To avoid any circularity, we evaluated the calibration by only keeping a single interaction par combination body sizes and predator present in the Barnes data set. When including all the interaction, the mean Boyce index value reached 0.64 ( $\mathrm{sd}=0.07, \mathrm{n}=999$ ), and when we kept only unique combination of predator- prey body sizes (mean Boyce index $=0.55$, $\mathrm{sd}=0.08, \mathrm{n}=999$ ).

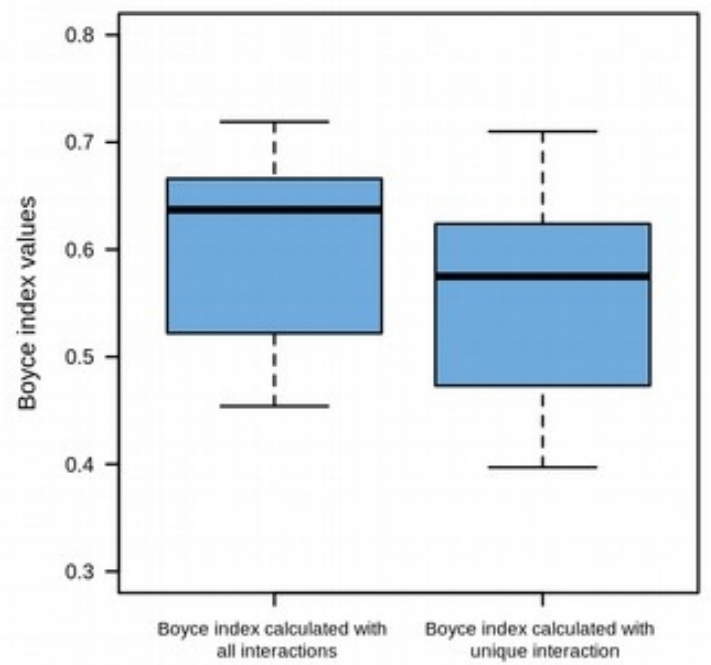


Fig. S3 | Maps of local food web indicators across the world ocean. The maps represent the distribution of, (a) omnivory index, (b) number of links, (c) number of top predators (d) number of basal species (e) number of intermediate species (f) vulnerability (mean number of consumer species per prey species), (g) generality (mean number of prey species per predator species), (h) modularity (i) percentage of shortest path length $<3$, (j) percentage of top predators (k) percentage of basal species and (l) percentage of intermediate species and for 11,365 fish species on a $1^{\circ} \times 1^{\circ}$ grid. The coastline was defined by using the Global Self-consistent, Hierarchical, High-resolution Geography Database (51).
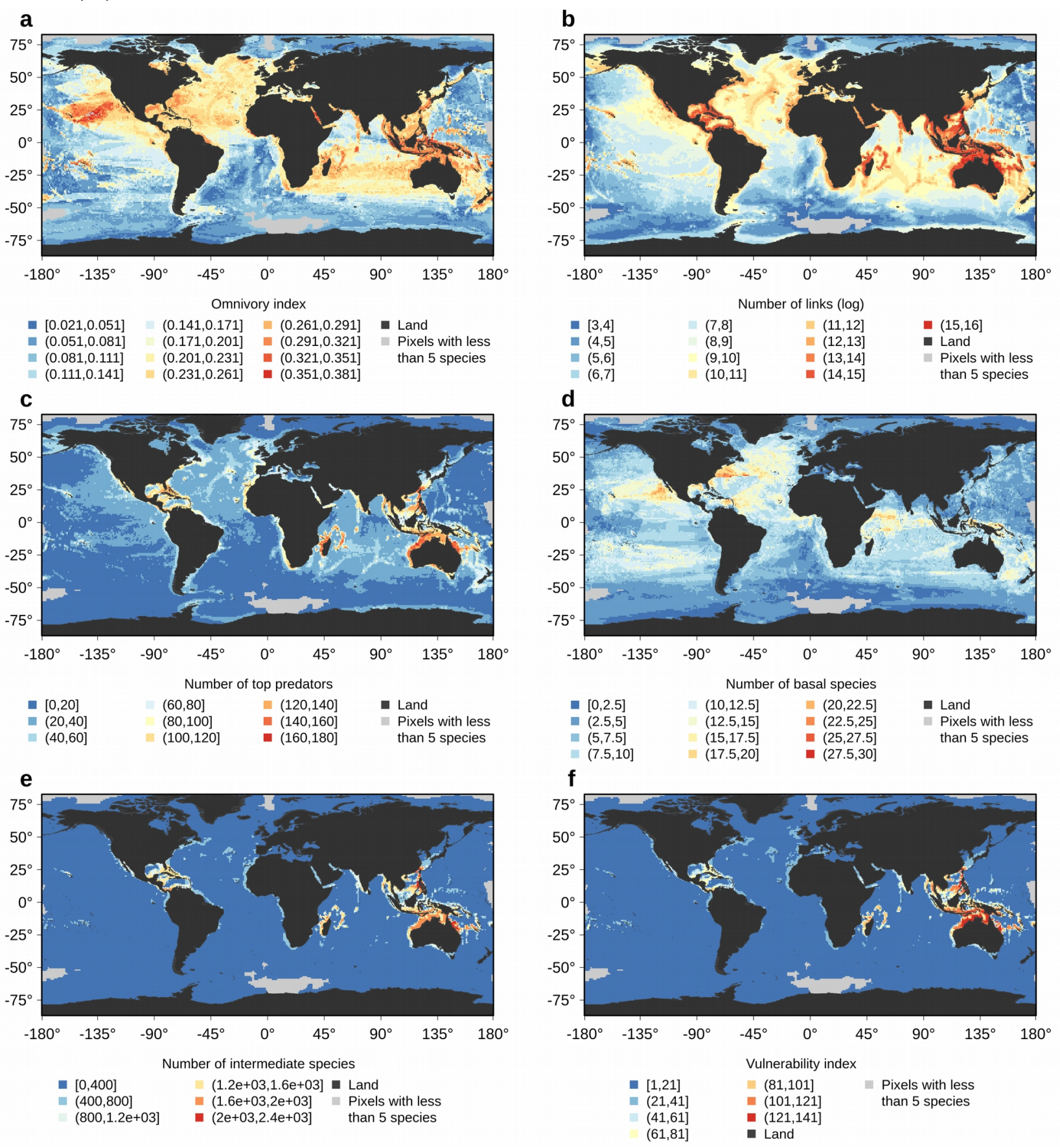

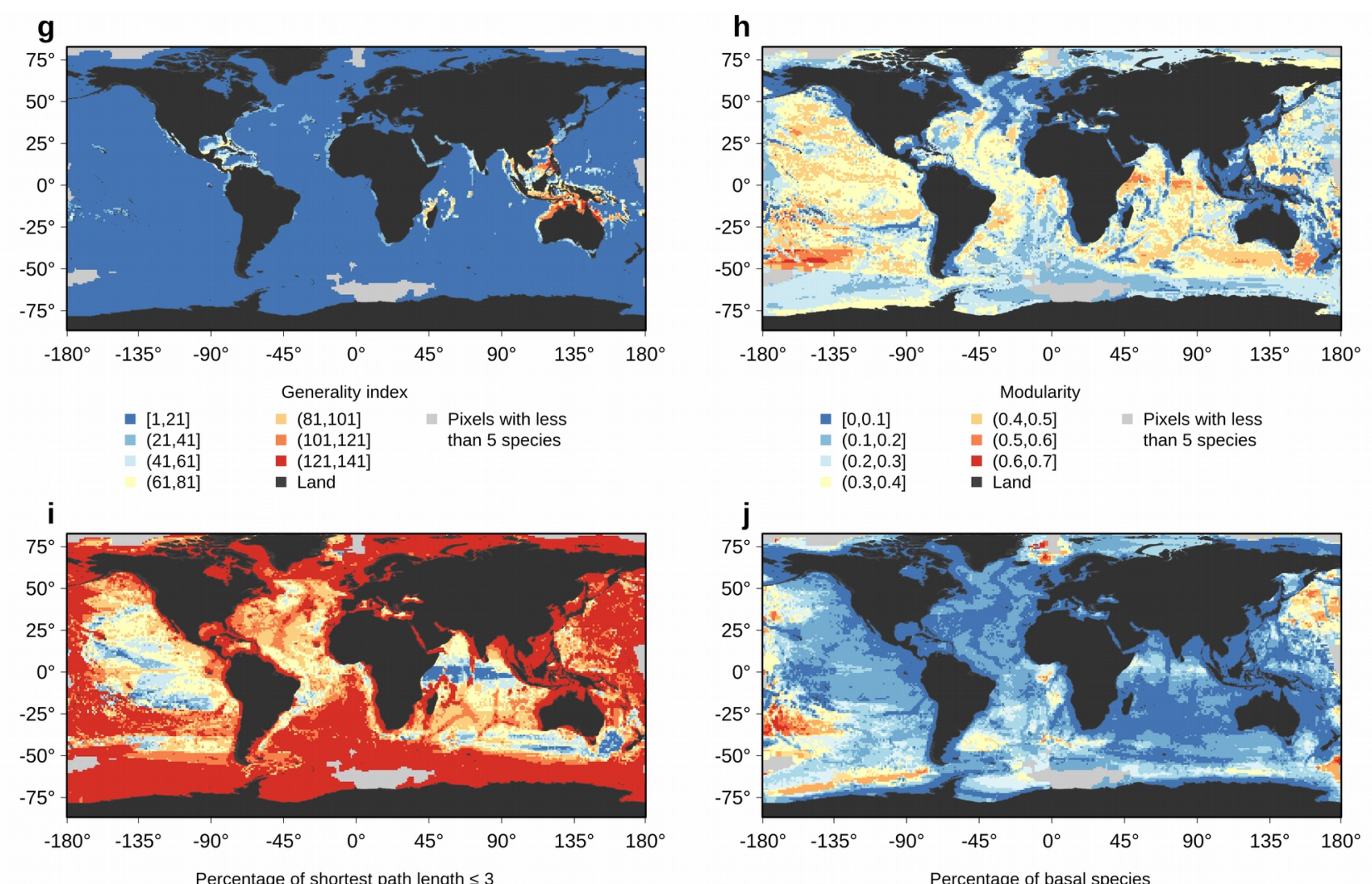

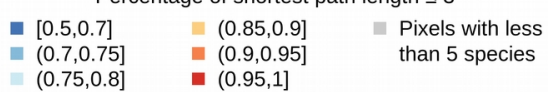

$$
\begin{aligned}
& (0.8,0.85] \text { Land }
\end{aligned}
$$

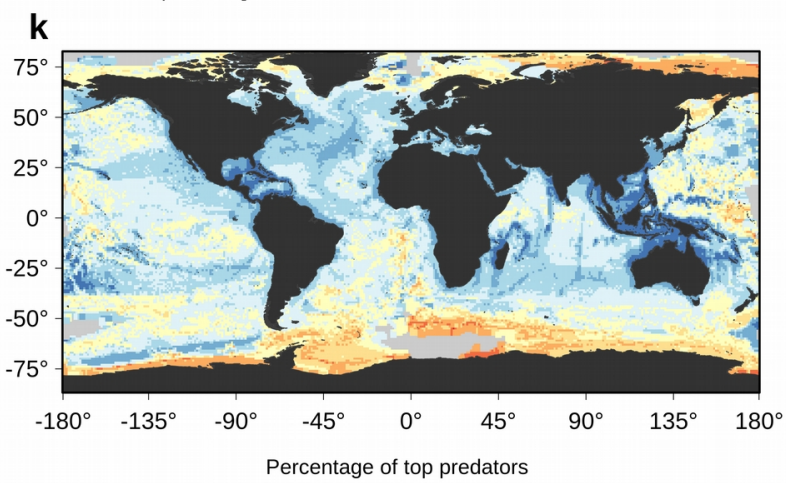

$$
\begin{array}{lll}
\square[0,10] & (40,50] & \\
\square(10,20] & (50,60] & \text { घand } \\
\square(20,30] & \square(60,70] & \text { Pixels with less } \\
(30,40] & \square(70,80] & \text { than } 5 \text { species }
\end{array}
$$

\begin{tabular}{|c|c|c|}
\hline$[0,10]$ & $(40,50]$ & ㅁ $(80,100]$ \\
\hline$(10,20]$ & $(50,60]$ & - Land \\
\hline$(20,30]$ & $\square(60,70]$ & Pixels with less \\
\hline$(30,40]$ & [ $(70,80]$ & than 5 species \\
\hline
\end{tabular}

\section{1}

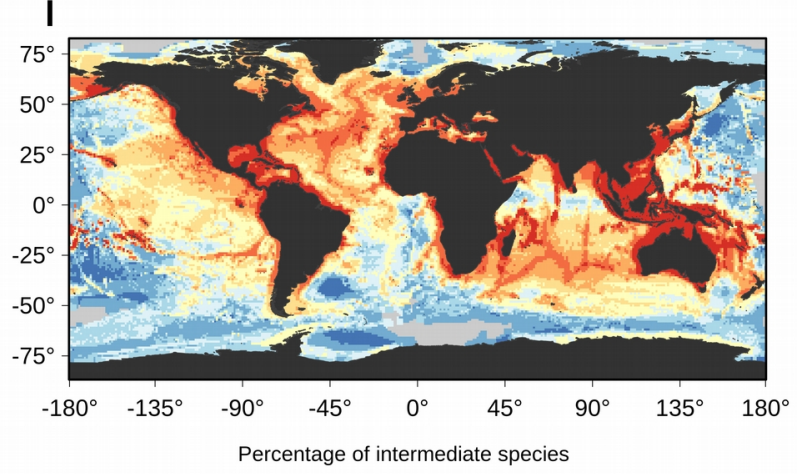


Fig. S4 |Maps of environmental descriptors. The maps represent the distribution of (a) mean primary productivity, (b) mean sea surface temperature, (c) mean sea surface salinity and (d) distance from land. For more details about the environmental descriptors, see table S2. The coastline was defined by using the Global Self-consistent, Hierarchical, High-resolution Geography Database (51).
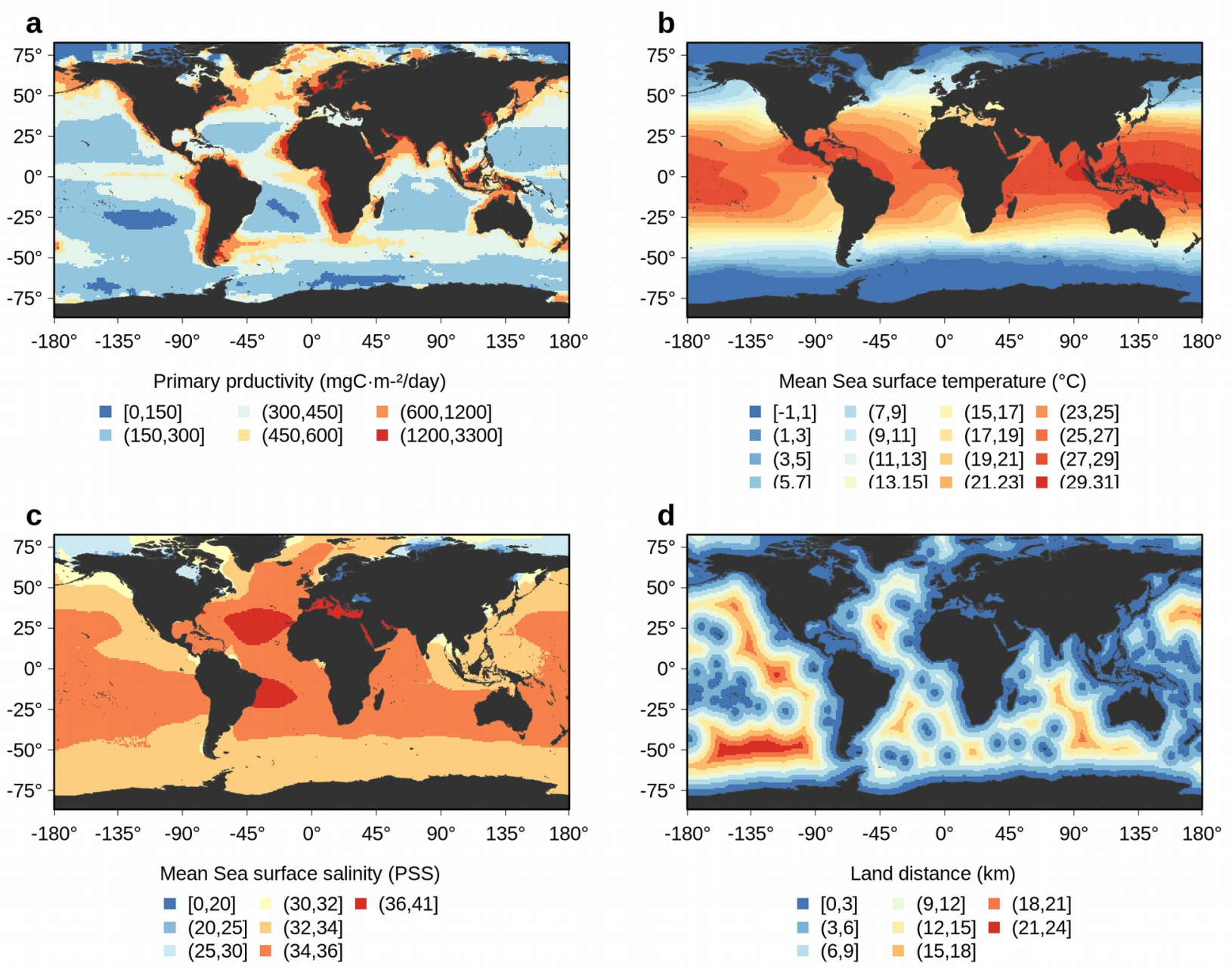
Fig. S5 | Partial spearman correlation matrix between estimated world trophic indicators and abiotic factors by excluding the effect of species richness. We added to this figure the spearman correlation between species richness and other indicators. The red colour indicates a significant negative correlation, while blue colours indicate a significant positive correlation between two trophic indicators. The colour gradient (from red to blue) indicates the magnitude of the correlation. White colour means that the correlation between indicators is not significant according to the Spearman correlation statistical test. (SST: Sea Surface Temperature; SSS: Sea Surface Salinity; Primary productivity; Distance to land). The coastline was defined by using the Global Selfconsistent, Hierarchical, High-resolution Geography Database (51).

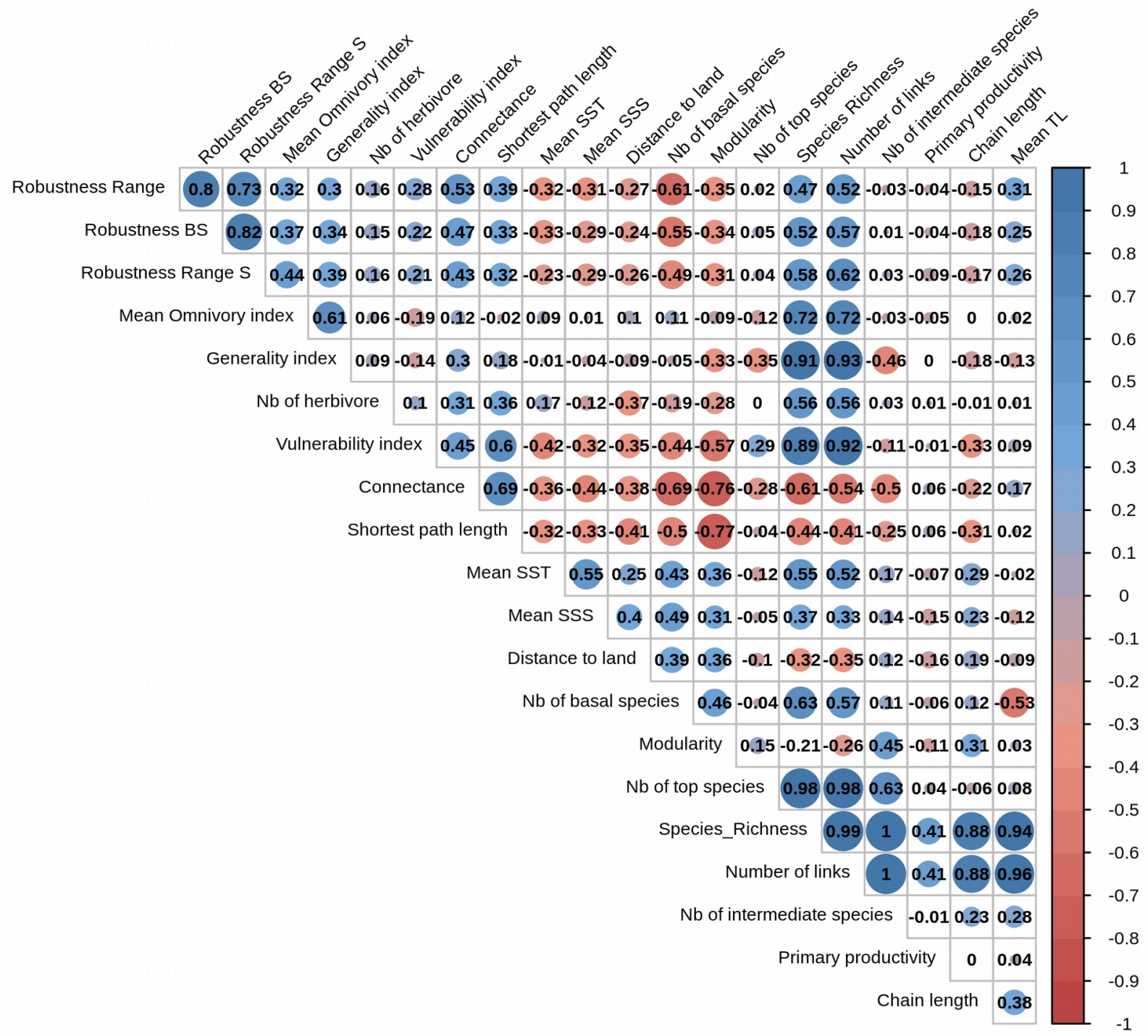


Fig. S6 | Spatial distribution of herbivorous species (a) on a $1^{\circ} \times 1^{\circ}$ grid and (b) averaged across latitudes. The GAM models were fitted between the mean number of herbivorous species (b; adjusted $\mathrm{R}^{2}=0.85$, edf $=8.8$ ), and latitude, within shaded grey the $95 \%$ confidence limits. The black bars on the chart represent the standard error of the mean. The coastline was defined by using the Global Self-consistent, Hierarchical, High-resolution Geography Database (51).

a

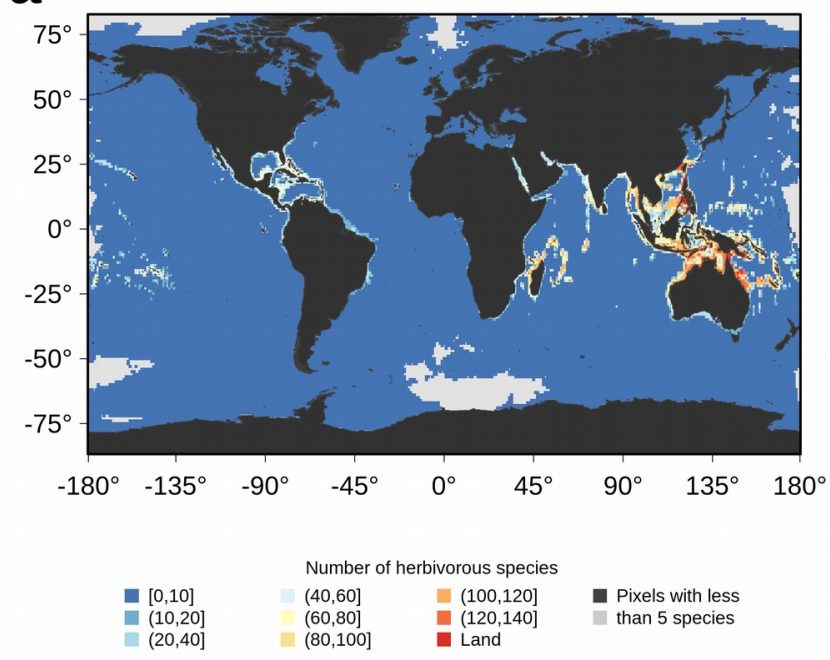

b

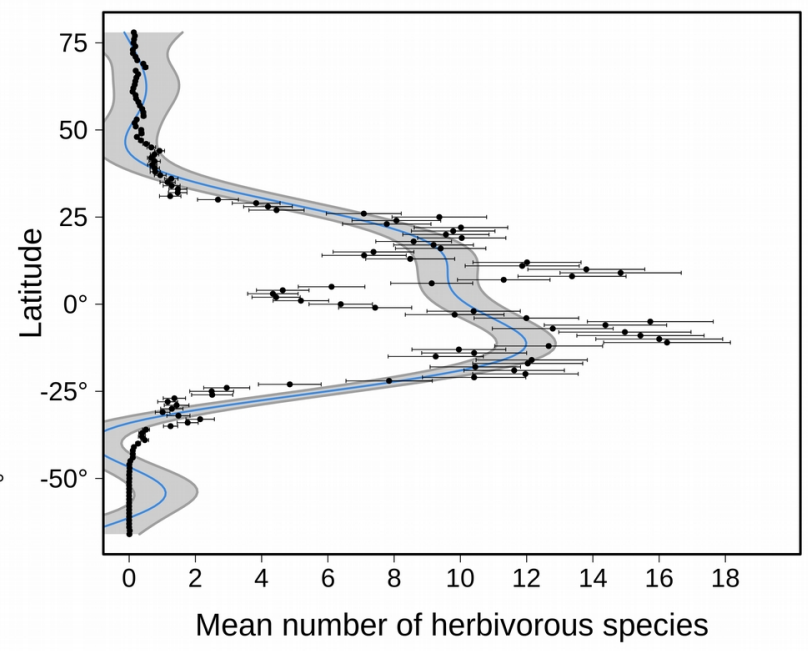


Fig. S7 | Map of local geometric mean body size (a) on a $1^{\circ} \times 1^{\circ}$ grid and (b) averaged across latitudes. The GAM models were fitted between the mean number of herbivorous species (b; adjusted $\mathrm{R}^{2}=0.96$, edf $=8.9$ ), and latitude, within shaded grey the $95 \%$ confidence limits. The black bars on the charts represent the standard error of the mean. The coastline was defined by using the Global Self-consistent, Hierarchical, High-resolution Geography Database (51).

a

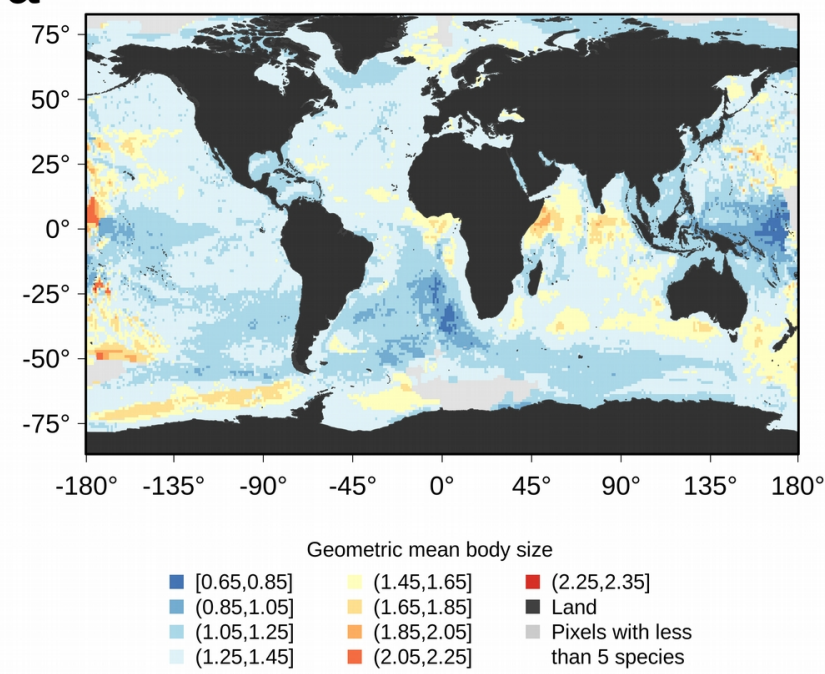

b

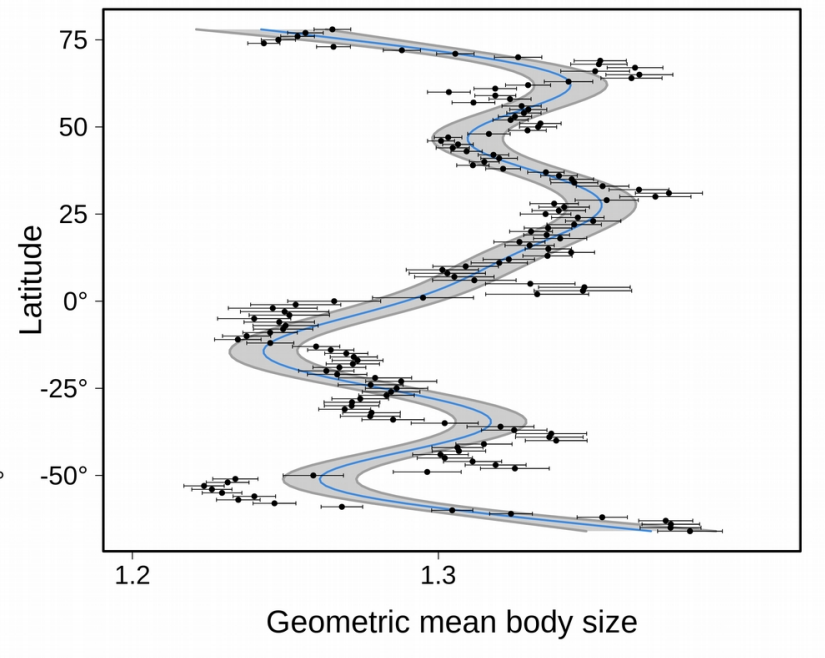


Fig. S8 | Spatial distribution of (a) the local food web robustness to random species extinctions and (b) its variance across the 99 scenarios. The coastline was defined by using the Global Selfconsistent, Hierarchical, High-resolution Geography Database (51).

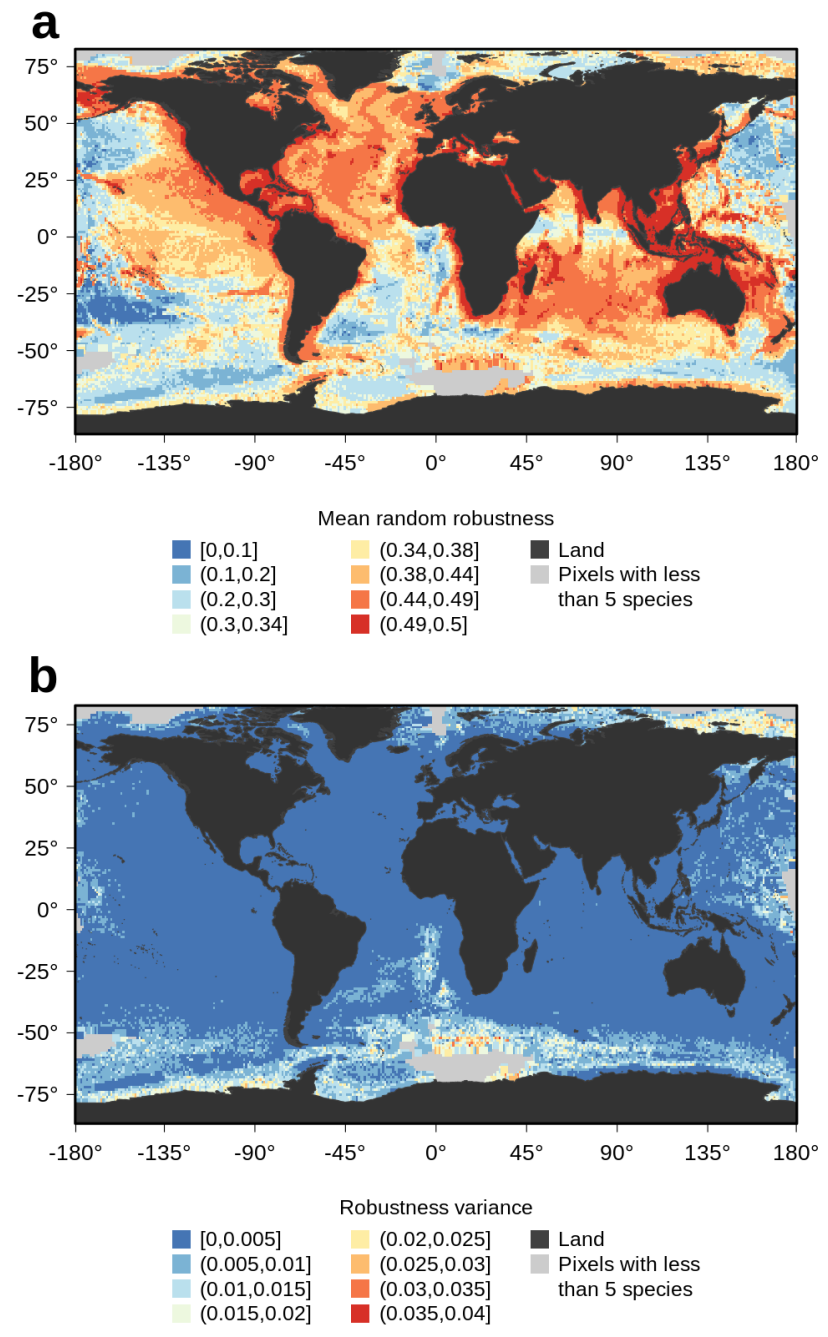


Fig. S9 | Representation of three different regression trees. (a) Relation between environmental factors (primary productivity, habitat, SST: sea surface temperature, salinity) and the robustness to species extinctions ordered by decreasing species body size. (b) Relation between environmental factors (primary productivity, distance from land, SST, salinity), species richness and the robustness to species extinctions ordered by decreasing species body size. The variable distance from land was transformed in a categorical variable, i.e. cells with a depth between $0-200 \mathrm{~m}$ were identified as coastal while the others as open ocean. (c) Relation between internal structure networks indicators and the robustness to species extinctions ordered by decreasing species body size. (d) Relation between internal structure networks indicators, species richness and the robustness to species extinctions ordered by decreasing species body size. We substituted the number of links by the species richness because these variables are highly correlated.

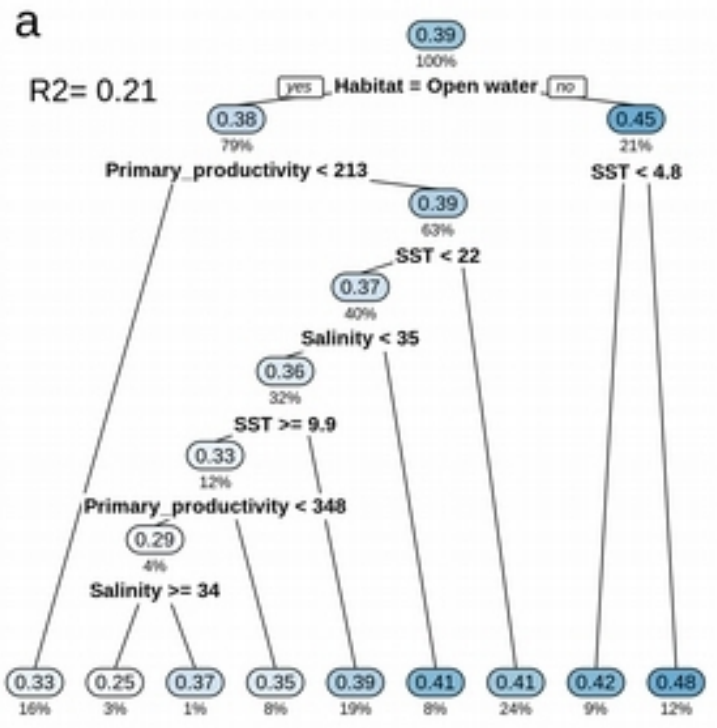

Robustness body size $\sim$ Habitat + Salinity + Primary productivity + SST

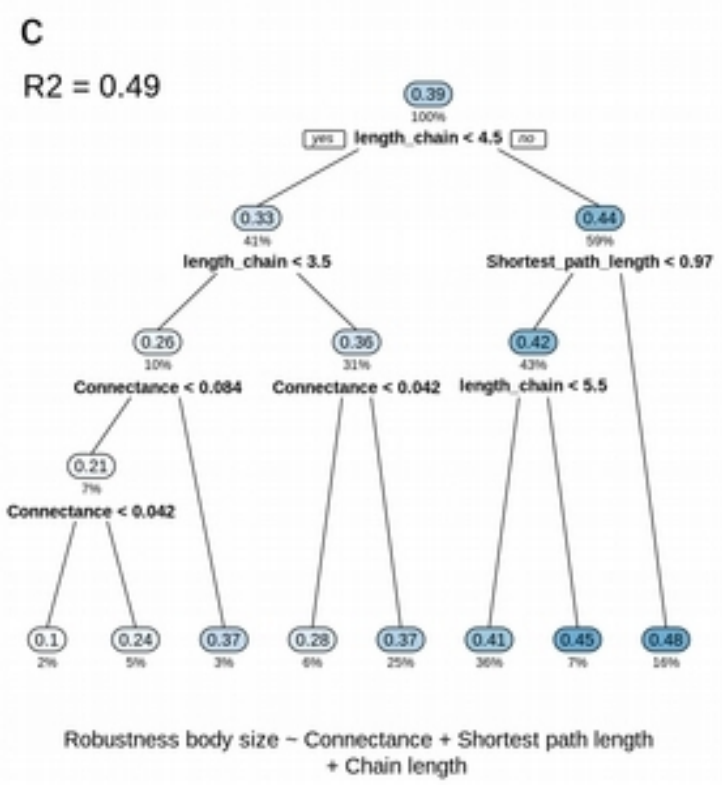

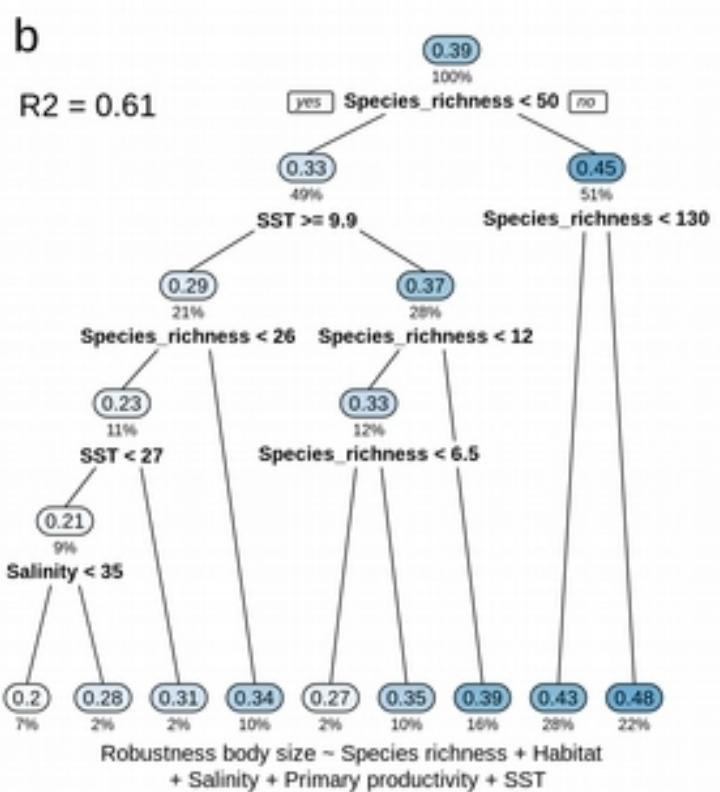

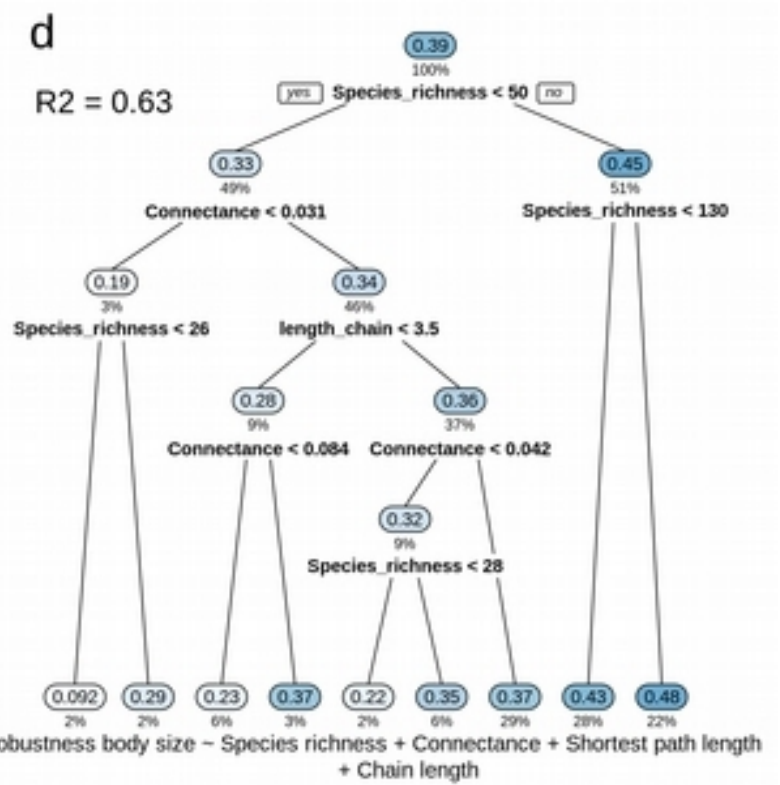


Fig S10 | Transformations conducted on the Ocean Biogeographic System OBIS data to obtain the fine scale species distribution. Here we use the distribution of the Mediterranean rainbow wrasse (Coris julis) as an example. Species data were obtained from the Ocean Biogeographic Information System (OBIS, http://www.iobis.org) on 08/27/2014. We reconstructed distribution maps for each species, defined as the convex polygon surrounding the area where each species was observed (see for details Fig. S3). The resulting polygon was divided into four parts across the world to integrate possible discontinuity between the two hemispheres and the Atlantic and Pacific Oceans. Then we refined each species distribution map by removing areas where maximal depths fell outside the minimum or maximum known depth range of the species (1). Bathymetry data were taken from ETOPO1 (2). Finally, we aggregated fish distributions on a $1^{\circ}$ resolution grid covering all oceans. All the data are freely available at https://figshare.com/s/c9ca229cc1f3548f8b5c. The coastline was defined by using the Global Self-consistent, Hierarchical, High-resolution Geography Database (51).

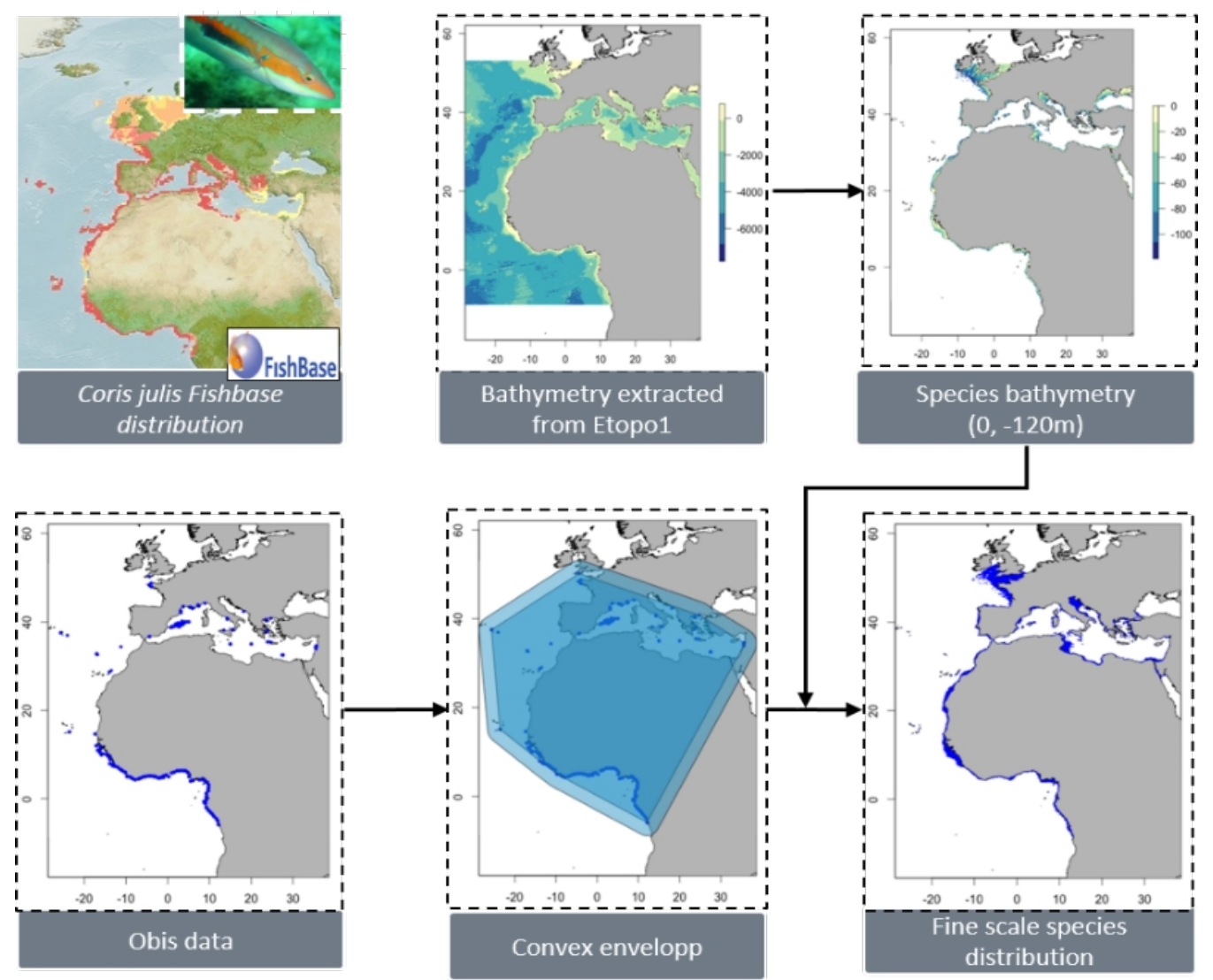

1. Froese, R. \& Pauly., D. Fishbase. FishBase World Wide Web electronic publication (2015). Available at: www.fishbase.org.

2. Amante, C. and B.W. Eakins. ETOPO1 1 Arc-Minute Global Relief Model: Procedures, Data Sources and Analysis. NOAA Technical Memorandum NESDIS NGDC-24. National Geophysical Data Center, NOAA. (2009). 
Table S1. Information on the 56 biogeochemical provinces (39).

\begin{tabular}{|c|c|c|c|}
\hline $\begin{array}{l}\text { Province } \\
\text { numbers }\end{array}$ & $\begin{array}{c}\text { Province } \\
\text { codes }\end{array}$ & Short provinces description & Climatic biomes \\
\hline 1 & BPLR & Polar - Boreal Polar Province (POLR) & Polar \\
\hline 2 & ARCT & Polar - Atlantic Arctic Province & Polar \\
\hline 3 & SARC & Polar - Atlantic Subarctic Province & Polar \\
\hline 4 & NADR & Westerlies - N. Atlantic Drift Province (WWDR) & Temperate \\
\hline 5 & GFST & Westerlies - Gulf Stream Province & Subtropical \\
\hline 6 & NASTW & Westerlies - N. Atlantic Subtropical Gyral Province (West) (STGW) & Subtropical \\
\hline 7 & NATR & Trades - N. Atlantic Tropical Gyral Province (TRPG) & Tropical \\
\hline 8 & WTRA & Trades - Western Tropical Atlantic Province & Equatorial \\
\hline 9 & ETRA & Trades - Eastern Tropical Atlantic Province & Equatorial \\
\hline 10 & SATL & Trades - South Atlantic Gyral Province (SATG) & Tropical \\
\hline 11 & NECS & Coastal - NE Atlantic Shelves Province & Temperate \\
\hline 12 & CNRY & Coastal - Canary Coastal Province (EACB) & Equatorial \\
\hline 13 & GUIN & Coastal - Guinea Current Coastal Province & Tropical \\
\hline 14 & GUIA & Coastal - Guianas Coastal Province & Equatorial \\
\hline 15 & NWCS & Coastal - NW Atlantic Shelves Province & Temperate \\
\hline 16 & MEDI & Westerlies - Mediterranean Sea, Black Sea Province & Subtropical \\
\hline 17 & CARB & Trades - Caribbean Province & Tropical \\
\hline 18 & NASTE & Westerlies - N. Atlantic Subtropical Gyral Province (East) (STGE) & Subtropical \\
\hline 19 & BRAZ & Coastal - Brazil Current Coastal Province & Tropical \\
\hline 20 & FKLD & Coastal - SW Atlantic Shelves Province & Temperate \\
\hline 21 & BENG & Coastal - Benguela Current Coastal Province & Subtropical \\
\hline 22 & MONS & Trades - Indian Monsoon Gyres Province & Tropical \\
\hline 23 & ISSG & Trades - Indian S. Subtropical Gyre Province & Subtropical \\
\hline 24 & EAFR & Coastal - E. Africa Coastal Province & Tropical \\
\hline 25 & REDS & Coastal - Red Sea, Persian Gulf Province & Tropical \\
\hline 26 & ARAB & Coastal - NW Arabian Upwelling Province & Tropical \\
\hline 27 & INDE & Coastal - E. India Coastal Province & Tropical \\
\hline 28 & INDW & Coastal - W. India Coastal Province & Tropical \\
\hline 29 & AUSW & Coastal - Australia-Indonesia Coastal Province & Subtropical \\
\hline 30 & BERS & Polar - N. Pacific Epicontinental Province & Polar \\
\hline 31 & PSAE & Westerlies - Pacific Subarctic Gyres Province (East) & Temperate \\
\hline 32 & PSAW & Westerlies - Pacific Subarctic Gyres Province (West) & Temperate \\
\hline 33 & KURO & Westerlies - Kuroshio Current Province & Subtropical \\
\hline 34 & NPPF & Westerlies - N. Pacific Polar Front Province & Subtropical \\
\hline 35 & NPSW & Westerlies - N. Pacific Subtropical Gyre Province (West) & Tropical \\
\hline 36 & TASM & Westerlies - Tasman Sea Province & Temperate \\
\hline 37 & SPSG & Westerlies - S. Pacific Subtropical Gyre Province & Subtropical \\
\hline 38 & NPTG & Trades - N. Pacific Tropical Gyre Province & Tropical \\
\hline 39 & PNEC & Trades - N. Pacific Equatorial Countercurrent Province & Equatorial \\
\hline 40 & PEQD & Trades - Pacific Equatorial Divergence Province & Equatorial \\
\hline 41 & WARM & Trades - W. Pacific Warm Pool Province & Equatorial \\
\hline 42 & ARCH & Trades - Archipelagic Deep Basins Province & Equatorial \\
\hline
\end{tabular}




\begin{tabular}{c|clc}
\hline 43 & ALSK & Coastal - Alaska Downwelling Coastal Province & Temperate \\
\hline 44 & CCAL & Coastal - California Upwelling Coastal Province & Subtropical \\
\hline 45 & CAMR & Coastal - Central American Coastal Province & Equatorial \\
\hline 46 & CHIL & Coastal - Chile-Peru Current Coastal Province & Tropical \\
\hline 47 & CHIN & Coastal - China Sea Coastal Province & Subtropical \\
\hline 48 & SUND & Coastal - Sunda-Arafura Shelves Province & Equatorial \\
\hline 49 & AUSE & Coastal - East Australian Coastal Province & Subtropical \\
\hline 50 & NEWZ & Coastal - New Zealand Coastal Province & Temperate \\
\hline 51 & SSTC & Westerlies - S. Subtropical Convergence Province & Polar \\
\hline 52 & SANT & Westerlies - Subantarctic Province & Polar \\
\hline 53 & ANTA & Polar - Antarctic Province & Polar \\
\hline 54 & APLR & Polar - Austral Polar Province & Subtropical \\
\hline 55 & NPSE & Northeast Pacific subtropical & Subtropical \\
\hline 56 & OCAL & California current &
\end{tabular}


Table S2 | Environmental variable descriptions

\begin{tabular}{|c|c|c|c|c|c|c|c|c|c|}
\hline Layers & Description & Unit & Type & $\begin{array}{c}\text { Spatial } \\
\text { Resolution }\end{array}$ & $\begin{array}{c}\text { Temporal } \\
\text { Range }\end{array}$ & Derivatives & Source & $\begin{array}{c}\text { Primary Data } \\
\text { Source }\end{array}$ & URL \\
\hline $\begin{array}{l}\text { Land } \\
\text { distance }\end{array}$ & $\begin{array}{l}\text { Distance }(\mathrm{km}) \text { to the nearest } \\
\text { land cell (water cells only) } \\
\text { calculated using Euclidean } \\
\text { distance formula using } \\
\text { ArcGIS. Values represented } \\
\text { in floating point integer. }\end{array}$ & $\mathrm{km}$ & - & 5 arcmin & - & - & - & - & $\begin{array}{l}\text { http://gmed.auckland.ac.nz/ } \\
\text { layersd.html }\end{array}$ \\
\hline Temperature & $\begin{array}{l}\text { Sea surface temperature is } \\
\text { the temperature of the water } \\
\text { at the ocean surface. This } \\
\text { parameter indicates the } \\
\text { temperature of the topmost } \\
\text { meter of the ocean water } \\
\text { column. }\end{array}$ & ${ }^{\circ} \mathrm{C}$ & $\begin{array}{l}\text { Monthly } \\
\text { climatolog } \\
\text { y }\end{array}$ & $\underset{\mathrm{km})}{\operatorname{arcmin}}(9.2$ & $2002-2009$ & Mean & Bio-Oracle & 1 & http://oceancolor.gsfc.nasa.gov/ \\
\hline Salinity & $\begin{array}{l}\text { Salinity indicates the } \\
\text { dissolved salt content in the } \\
\text { ocean surface. }\end{array}$ & PSS & $\begin{array}{c}\text { In situ } \\
\text { measure: } \\
\text { WOD } 2009\end{array}$ & $1^{\circ} \times 1^{\circ}$ & $1961-2009$ & Mean & Bio-Oracle & 2 & http://www.nodc.noaa.gov/ \\
\hline $\begin{array}{l}\text { Primary } \\
\text { productivity }\end{array}$ & $\begin{array}{l}\text { Proportion of annual primary } \\
\text { production in a cell. See } \\
\text { reference for details about } \\
\text { the productivity calculation } \\
\text { methods. }\end{array}$ & $\begin{array}{l}\mathrm{mgC} \cdot \\
\mathrm{m}-2 \cdot / \\
\text { day/ } \\
\text { cell }\end{array}$ & $\begin{array}{c}\text { Annual } \\
\text { climatolog } \\
y\end{array}$ & $\begin{array}{l}5 \underset{\mathrm{km}}{\operatorname{arcmin}}(9 \\
\end{array}$ & - & Mean & $\begin{array}{l}\text { Aquamaps } \\
\text { HCAF v4 }\end{array}$ & $3,4,5$ & $\begin{array}{c}\text { http://www.aquamaps.org/download/ } \\
\text { main.php and http://www.seaaroundus.org/ } \\
\text { doc/saup_manual.htm\#3 }\end{array}$ \\
\hline
\end{tabular}

1. Feldman, G.C., C.R. McClain, N. Kuring (ed.), S.W. Bailey (ed.). 2006. Sea-viewing Wide Field-of-View Sensor (SeaWIFS) Level-3 Standard Mapped Images: Chlorophyll-a Mean Concentration Annual and Seasonal Climatologies. NASA Goddard Space Flight Center

2. $\quad$ Boyer, T. P., Stephens, C., J. I. Antonov, M. E. Conkright, R. A. Locarnini, T. D. O’Brien, H. E. Garcia, 2002: World Ocean Atlas 2001, Volume 2:

Salinity. S. Levitus, Ed., NOAA Atlas NESDIS 50, U.S. Government Printing Office, Wash., D.C., 165 pp., CD-ROMs.

3. Bouvet, M., Hoepffner, N. \& Dowell, M. D. Parameterization of a spectral solar irradiance model for the global ocean using multiple satellite sensors. J. Geophys. Res. Ocean. 107, 8-1-8-18 (2002).

4. Hoepffner, N., Sturm, B., Finenko, Z. \& Larkin, D. Depth-integrated primary production in the eastern tropical and subtropical North Atlantic basin from ocean colour imagery. Int. J. Remote Sens. 20, 1435-1456 (1999).

5. Longhurst, A., Sathyendranath, S., Platt, T. \& Caverhill, C. An estimate of global primary production in the ocean from satellite radiometer data. J. Plankton Res. 17, 1245-1271 (1995). 
Table S3.Spearman correlations between the four sequential removal scenarios. The body size scenario corresponds to the removing of species by decreasing species body size. The largest range size scenario corresponds to species extinctions ordered by decreasing species range size. The smallest range size scenario corresponds to species extinctions ordered by increasing species range size. The random scenario was obtained by removing the species randomly (99 random deletion sequences initiated for each web). All correlations were significant $(\mathrm{P}<0.001)$.

\begin{tabular}{|c|c|c|c|c|}
\cline { 2 - 5 } \multicolumn{1}{c|}{} & Body size scenario & $\begin{array}{c}\text { Largest range size } \\
\text { scenario }\end{array}$ & $\begin{array}{c}\text { Smallest range size } \\
\text { scenario }\end{array}$ & Random scenario \\
\hline $\begin{array}{c}\text { Body size } \\
\text { scenario }\end{array}$ & 1 & 0.86 & 0.88 & 0.88 \\
\hline $\begin{array}{c}\text { Largest range } \\
\text { size scenario }\end{array}$ & 0.86 & 1 & 0.82 & 0.86 \\
\hline $\begin{array}{c}\text { Smallest range } \\
\text { size scenario }\end{array}$ & 0.88 & 0.82 & 1 & 0.93 \\
\hline Random scenario & 0.88 & 0.86 & 0.93 & 1 \\
\hline
\end{tabular}

Table S4 | Wilcoxon rank tests performed between mean coastal and open water indicators. Values for robustness referred to the sequential removal scenario of the largest-bodied size species (Rob BS), the smallest-bodied size species (Rob Range small) and the species with the largest range (Rob Range). SST referred to the Sea Surface Temperature and the SSS referred to the Sea Surface Salinity.

\begin{tabular}{cccccc}
\hline Indicators & $\begin{array}{c}\text { Coastal } \\
\text { Mean }\end{array}$ & $\begin{array}{c}\text { Coastal } \\
\text { sd }\end{array}$ & $\begin{array}{c}\text { Open water } \\
\text { Mean }\end{array}$ & $\begin{array}{c}\text { Open water } \\
\text { sd }\end{array}$ & p-value \\
\hline Rob BS & 0.45 & 0.06 & 0.38 & 0.1 & $<0.001$ \\
\hline Rob Range & 0.46 & 0.06 & 0.38 & 0.1 & $<0.001$ \\
\hline $\begin{array}{c}\text { Rob Range } \\
\text { small }\end{array}$ & 0.46 & 0.06 & 0.38 & 0.11 & $<0.001$ \\
\hline $\begin{array}{c}\text { Species } \\
\text { richness }\end{array}$ & 366.95 & 499.35 & 66.10 & 120.11 & $<0.001$ \\
\hline Path length & 4.97 & 0.92 & 4.22 & 1.15 & $<0.001$ \\
\hline $\begin{array}{c}\text { Number of } \\
\text { links }\end{array}$ & $21,404.58$ & $48,048.7$ & 824 & 5910.34 & $<0.001$ \\
\hline Connectance & 0.06 & 0.03 & 0.09 & 0.13 & $<0.001$ \\
\hline Modularity & 0.13 & 0.12 & 0.28 & 0.11 & $<0.001$ \\
\hline $\begin{array}{c}\text { Omnivory } \\
\text { index }\end{array}$ & 0.17 & 0.08 & 0.16 & 0.08 & $<0.001$ \\
\hline Generality & 20.05 & 29.09 & 3.34 & 5.43 & $<0.001$ \\
\hline $\begin{array}{c}\text { Primary } \\
\text { productivity }\end{array}$ & 545.35 & 426.79 & 314.79 & 166.74 & $<0.001$ \\
\hline SST & 11.7 & 11.91 & 14.1 & 10.83 & $<0.001$ \\
\hline SSS & 32 & 4.36 & 34 & 1.65 & $<0.001$ \\
\hline
\end{tabular}


Table S5 | Results of generalized linear models with a Gaussian distribution for continuous explicated variables and quasi-Poisson distribution for discretionary explicated variables. We included as predictors the coastal or open ocean factor and the species richness. The sign of the t-statistic indicate which mean is greater (open water versus coastal) and the p-value of the factor terms indicate if the difference is significative accounting for the species richness. Finaly, we performed a Moran's I test on the residual of the glm model to test the spatial autocorrelation.

\begin{tabular}{|c|c|c|c|c|c|}
\hline \multirow[t]{2}{*}{ Connectance } & \multirow[b]{2}{*}{ Estimate } & \multirow[b]{2}{*}{ t value } & \multicolumn{3}{|l|}{ Generality } \\
\hline & & & & Estimate & t value \\
\hline Intercept & 0.083 & $53.02 * * *$ & Intercept & 1.66 & $231.81^{* * *}$ \\
\hline Open ocean & 0.018 & $11.41 * * *$ & Open ocean & -0.62 & $-78.07 * * *$ \\
\hline \multirow[t]{2}{*}{ Species richness } & $-6.07 e-05$ & $-26.03^{* * *}$ & Species richness & 0.0019 & $359.89 * * *$ \\
\hline & Expected & Observed & & Expected & Observed \\
\hline Moran’s I & $-2.32 e-05$ & $0.15^{* * *}$ & Moran’s I & $-2.32 \mathrm{e}-05$ & $0.079 * * *$ \\
\hline \multirow[t]{2}{*}{ Link } & & & Modularity & & \\
\hline & Estimate & t value & & Estimate & t value \\
\hline Intercept & 7.65 & $696.02 * * *$ & Intercept & 0.19 & $134.10^{* * *}$ \\
\hline Open ocean & -1.23 & $-86.37 * * *$ & Open ocean & 0.10 & $68.73 * * *$ \\
\hline \multirow[t]{2}{*}{ Species richness } & 0.0026 & $384.57 * * *$ & Species richness & -0.00016 & $-75.01^{* * *}$ \\
\hline & Expected & Observed & & Expected & Observed \\
\hline Moran’s I & $-2.32 \mathrm{e}-05$ & $0.066^{* * *}$ & Moran’s I & $-2.32 e-05$ & $0.078 * * *$ \\
\hline \multirow[t]{2}{*}{ Omnivory index } & & & Path length & & \\
\hline & Estimate & t value & & Estimate & t value \\
\hline Intercept & -2.04 & $-315^{* * *}$ & Intercept & 1.45 & $467.65^{* * *}$ \\
\hline Open ocean & 0.17 & $25.29 * * *$ & Open ocean & -0.038 & $-11.75^{* * *}$ \\
\hline \multirow[t]{2}{*}{ Species richness } & 0.00065 & $90.14^{* * *}$ & Species richness & 0.00036 & $92.07 * * *$ \\
\hline & Expected & Observed & & Expected & Observed \\
\hline Moran’s I & $-2.32 e-05$ & $0.12 * * *$ & Moran’s I & $-2.32 e-05$ & $0.14^{* * *}$ \\
\hline \multirow[t]{2}{*}{ Robustness body size } & & & Robustness range & & \\
\hline & Estimate & t value & & Estimate & t value \\
\hline Intercept & 0.41 & $330.85 * * *$ & Intercept & 0.42 & $342 * * *$ \\
\hline Open ocean & -0.04 & $-31.16^{* * *}$ & Open ocean & -0.047 & $-36.76^{* * *}$ \\
\hline \multirow[t]{2}{*}{ Species richness } & 0.0001 & $55.41^{* * *}$ & Species richness & $9.87 e-05$ & $53.27 * * *$ \\
\hline & Expected & Observed & & Expected & Observed \\
\hline Moran’s I & $-2.49 e-05$ & $0.11^{* * *}$ & Moran’s I & $-2.49 e-05$ & $0.10 * * *$ \\
\hline \multicolumn{6}{|l|}{ Robustness small range } \\
\hline & Estimate & t value & & & \\
\hline Intercept & 0.41 & $314.97 * * *$ & & & \\
\hline Open ocean & -0.045 & $-33.43 * * *$ & & & \\
\hline \multirow[t]{2}{*}{ Species richness } & 0.00012 & $58.56 * * *$ & & & \\
\hline & Expected & Observed & & & \\
\hline Moran’s I & $-2.49 e-05$ & $0.12 * * *$ & & & \\
\hline
\end{tabular}

